\title{
Effects of delayed treatment with nebracetam on neurotransmitters in brain regions after microsphere embolism in rats
}

\author{
${ }^{1}$ Satoshi Takeo, Hideki Hayashi, Keiko Miyake, Kaori Takagi, Mina Tadokoro, Norio Takagi \& \\ Sayuri Oshikawa
}

Department of Pharmacology, Tokyo University of Pharmacy \& Life Science, Hachioji, Tokyo, 192-03 Japan

1 The effects of delayed treatment with nebracetam, a novel nootropic drug, on neurotransmitters of brain regions were examined in rats with microsphere embolism-induced cerebral ischaemia.

2 Cerebral ischaemia was induced by administration of 900 microspheres $(48 \mu \mathrm{m})$ into the internal carotid artery. The rats with stroke-like symptoms were treated p.o. with $30 \mathrm{mg} \mathrm{kg}{ }^{-1}$ nebracetam twice daily. The levels of acetylcholine, dopamine, noradrenaline, 5-hydroxytryptamine (5-HT) and their metabolites in the cerebral cortex, striatum and hippocampus of animals with microsphere embolism were determined by high performance liquid chromatography (h.p.l.c.) on the 3rd and 7th days after the operation.

3 Although the microsphere embolism induced significant changes in most of the neurotransmitters and some of their metabolites in the brain regions, the delayed treatment with nebracetam partially restored only the hippocampal 5-HT and the striatal dopamine metabolite contents on the 3rd day.

4 The hippocampal in vivo 5-HT synthesis, but not the striatal dopamine synthesis, was attenuated in rats with microsphere embolism on the 3 rd day, but was restored by treatment with nebracetam. In vivo striatal dopamine turnover rate of the rats with microsphere embolism was inhibited on the 3rd day irrespective of treatment with nebracetam.

5 The present study provides evidence for a possible action of nebracetam on 5-HT metabolism in the ischaemic brain.

Keywords: Cerebral ischaemia; dopamine; microsphere embolism; nebracetam; 5-hydroxytryptamine

\section{Introduction}

Clinical studies have shown that nebracetam, 4-aminomethyl1-benzylpyrrolidin-2-one hemifumarate, is a possible therapeutic agent for patients with sequelae of cerebral infarction and cerebral haemorrhage with psychiatric symptoms (Ohtomo et al., 1992). Several pharmacological studies in the experimental animals have demonstrated that this agent improves energy metabolism such as glucose uptake in brain slices impaired by exposure to excess L-glutamate or hypoxia/ hypoglycaemia (Shibata et al., 1992) and protects high energy phosphate breakdown in the ischaemic brain of the rabbit (Kuhn et al., 1988). In addition to the improvement of energy metabolism, this agent has also been suggested to have a cholinomimetic action. This is supported by data from several behavioural studies, which include improvements of choline toxin-induced reduction in latency of the passive avoidance response (Hashimoto et al., 1991) and scopolamine-induced memory loss (Kuhn et al., 1988), and disruption of spatial cognition (Iwasaki et al., 1992) in rats. This agent has also been shown to attenuate scopolamine-induced decrease in catecholamine content in the thalamus and hypothalamus (Iwasaki et al., 1992). These findings suggest that nebracetam modulates neurotransmitters which play a key role in neurotransmission under pathophysiological conditions. However, whether the agent may affect neurotransmitter metabolism in the ischaemic brain remains unclear.

The experimental evidence for the effects of nebracetam described so far has been demonstrated largely in animals pretreated with this agent or tissues treated with the agent under in vitro conditions. Since most therapeutic agents are primarily administered to patients after the setting of, or

\footnotetext{
${ }^{1}$ Author for correspondence at: Department of Pharmacology, Tokyo University of Pharmacy \& Life Science, 1432-1, Horinouchi, Hachioji, Tokyo 192-03 Japan.
}

during the pathogenesis of disease, delayed treatment may be more relevant to the clinical use of such drugs. The present study was designed to examine whether or not delayed treatment with nebracetam affected neurotransmitter metabolism in the ischaemic brain.

Microsphere embolism was used in the present study to induce ischaemic damage to the brain because this model reveals typical features of cerebral ischaemia in rats, such as a reduction in cerebral blood flow (Miyake et al., 1993) and decrease in tissue high energy phosphate (Takeo et al., 1992), acetylcholine (Taguchi et al., 1994) and monoamines (Takagi et al., 1996) and, eventually, induces a $40-60 \%$ cerebral infarction in the ipsilateral hemisphere (Miyake et al., 1993).

\section{Methods}

\section{Preparation of animals}

Male Wistar rats weighing 180 to $220 \mathrm{~g}$ (Charles River Japan Inc., Atsugi, Japan) were used in the present study. The animals were maintained under artificial conditions at $23 \pm 1{ }^{\circ} \mathrm{C}$ with a constant humidity of $55 \pm 5 \%$ with a cycle of $12 \mathrm{~h} \mathrm{light}$ and $12 \mathrm{~h}$ dark, and had free access to food and tap water according to the Guideline of Experimental Animal Care issued by the Prime Minister's Office of Japan. This experimental protocol was approved by the University Committee of Animal Care and Welfare.

Microsphere embolism was performed by the method previously described (Miyake et al., 1989). Briefly, the rats were anaesthetized with $35 \mathrm{mg} \mathrm{kg}^{-1}$ sodium pentobarbitone, i.p., and fixed in a supine position on an operation plate. After cervical incision, the right common carotid artery was isolated. The right external carotid and the right pterygopalatine arteries were ligated with thread. Nine hundred microspheres 
$(47.5 \pm 0.5 \mu \mathrm{m}$ in diameter; NEN-005, New England Nuclear Inc., Boston, MA, U.S.A.) suspended in $20 \%$ dextran were injected into the right internal carotid artery through a polyethylene catheter placed in the right common carotid artery. The wound was closed and the animals were allowed to recover under artificial conditions as described above. The rats which underwent sham operation were injected with the same volume of vehicle without microspheres.

\section{Neurological deficits}

Fifteen hours after the operation, behaviour of the operated rats was scored on the basis of paucity of movement, truncal curvature and forced circling during locomotion, which were considered to be typical symptoms of stroke in rats (Furlow \& Bass, 1976; McGraw, 1977). The score of each feature was ranked from 3 to 0 (3, very severe; 2 , severe; 1 , moderate; 0 , light or no symptoms). The rats which had more than 6 points were considered to be type A, 6 to 4 type B and less than 4 type C. The symptoms and survival rate of the operated animals were similar to those previously described (Miyake et al., 1989; Takeo et al., 1992); for example, the symptoms of type A, B and $\mathrm{C}$ determined on the 1 st day were 54,10 and $3 \%$, respectively, and the mortality of the operated rats by the 1 st day after the operation was $33 \%$. In the present study, only the type A animals were used for the following study.

\section{Treatment with nebracetam}

After stroke-like symptoms of the microsphere-injected rats had been determined, the type A animals were randomly divided into two groups, nebracetam-treated and untreated groups. Nebracetam, $30 \mathrm{mg} \mathrm{kg}^{-1}$ was administered orally, twice daily to one group, whereas vehicle (water) was administered to the other group. This treatment was conducted until the 3 rd or 7 th days after the operation. Thus, the animals examined on the 3 rd and 7 th days after the operation received 5 and 13 doses of this agent, respectively. The dose of nebracetam used in the present study was chosen on the basis of the dose-response effect of this agent ranging from 10 to $100 \mathrm{mg} \mathrm{kg}^{-1}$ twice daily on the cerebral energy metabolism in microsphere-embolized rats in a previous study (Takeo et al., 1997).

\section{Measurement of acetylcholine (ACh) and monoamines in the brain regions}

Measurement of ACh and monoamines was performed by the method described previously (Taguchi et al., 1994; Takagi et al., 1996). Briefly, at an appropriate time in the experimental sequence, the microsphere-injected, sham-operated and nonoperated (pre-operated) rats were killed by focal microwave irradiation of the head with a strength of $5 \mathrm{~kW}$ for $0.85 \mathrm{~s}$ by a microwave applicator (TMW-6402C, Muromachi Kikai Co., Tokyo). After decapitation, the head of the animal was immersed into liquid nitrogen and left for $10 \mathrm{~s}$ (near freezing). The cerebral hemispheres were isolated and three brain regions, cerebral cortex, striatum and hippocampus, were separated. In previous studies, we observed that there was little or no pathophysiological alteration of the left hemisphere of the brain in this model (Takeo et al., 1992; Miyake et al., 1993; Taguchi et al., 1994; Takagi et al., 1996). Thus, we examined metabolic alterations in the brain regions of only the right hemisphere in the present study. Each brain region was homogenized in $0.2 \mathrm{M} \mathrm{HClO}_{4}$ and $0.01 \%$ ethylenediaminetetraacetic acid (EDTA) containing $1 \mu \mathrm{M}$ ethylhomocholine as an internal standard for $\mathrm{ACh}$ and choline. The homogenate was centrifuged at $10000 \mathrm{xg}$ for $15 \mathrm{~min}$ at $4^{\circ} \mathrm{C}$. The supernatant fluid was filtered through a membrane filter $(0.45 \mu \mathrm{M})$ and applied to high-performance liquid chromatography system with an electrochemical detector h.p.l.c.-e.c.d.; ECD-300, Eicom, Kyoto, Japan) to determine the concentrations of ACh, dopamine, 3,4-dihydroxyphenylacetic acid (DOPAC), homovanillic acid (HVA), noradrenaline (NA), 5-hydroxytryptamine (5-HT) and 5-hydroxyindoleacetic acid (5HIAA). For determination of ACh and choline, the sample was applied to a column consisting of Eicompak AC-gel and AC enzympak (Eicom, Kyoto, Japan) with the mobile phase containing $0.1 \mathrm{M} \mathrm{Na}_{2} \mathrm{HPO}_{4}, 200 \mathrm{mg}^{-1}$ sodium 1-decanesulphonate and $65 \mathrm{mg}^{-1}$ tetraethylammonium chloride, $\mathrm{pH} 8.5$, at a flow rate of $1 \mathrm{ml} \mathrm{min}{ }^{-1}$ at $33^{\circ} \mathrm{C}$. For determination of monoamines and their metabolites, the sample was applied to a reverse phase column (MA-5ODS, Eicom, Kyoto, Japan) with the mobile phase containing $0.1 \mathrm{M}$ citric acid, $0.1 \mathrm{M}$ sodium acetate, $5 \mathrm{mg}^{-1}$ disodium EDTA, $230 \mathrm{mg} \mathrm{l}^{-1}$ sodium

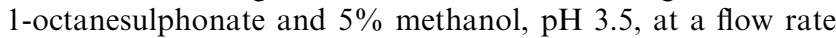
of $1 \mathrm{ml} \mathrm{min}{ }^{-1}$ at $25^{\circ} \mathrm{C}$.

\section{Determination of in vivo tyrosine and tryptophan hydroxylation}

To examine changes in the in vivo synthesis of monoamines, tyrosine or tryptophan hydroxylation, a rate limiting step for synthesis of each monoamine, was estimated by measuring the ex vivo accumulation of dopa or 5-hydroxytrypophan (5-HTP), respectively, after inhibition of aromatic L-amino acid decarboxylase with 3-hydroxybenzylhydrazine dihydrochloride (NSD-1015) as described earlier (Takagi et al., 1995). Three hours or more after the last administration of nebracetam, the microsphere-injected and sham-operated animals were given $100 \mathrm{mg} \mathrm{kg} \mathrm{kg}^{-1}$ NSD-1015, i.p., on the 3rd day after the operation. Thirty minutes after the administration of the inhibitor, the head of the rat was irradiated with a $5 \mathrm{~kW}$ microwave followed by near freezing in liquid nitrogen as described above. The right hemisphere was isolated and the striatum and hippocampus were separated. The dopa and 5-HTP were extracted from each brain region and analysed by h.p.l.c.-e.c.d. The extract was applied to the column for monoamines (MA-5ODS, Eicom, Kyoto, Japan) with the mobile phase containing $0.1 \mathrm{M}$ citric acid, $0.1 \mathrm{M}$ sodium acetate, $\mathrm{pH} 3.5,15 \%$ methanol, $240 \mathrm{mg} \mathrm{l}^{-1}$ sodium 1-octanesulphate, $5 \mathrm{mg} \mathrm{l}^{-1}$ disodium

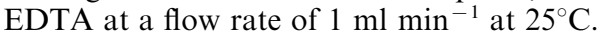

\section{Determination of in vivo monoamine catabolism rate}

In vivo dopamine turnover rate (catabolic rate) was determined by the method described previously (Takagi et al., 1995). The microsphere-injected and sham-operated rats were treated with $250 \mathrm{mg} \mathrm{kg}^{-1} \alpha$-methyl-p-tyrosine (i.p.), a tyrosine hydroxylase inhibitor, in saline on the 3rd day after the operation. Four hours later the head of the animal was irradiated with a $5 \mathrm{~kW}$ microwave as described above followed by the near freezing in liquid nitrogen. Dopamine was extracted from the striatum and determined by h.p.l.c.-e.c.d. as described above. The sample was applied to a column of CA-5ODS (Eicom, Kyoto, Japan) with the mobile phase containing $0.1 \mathrm{M} \mathrm{NaH}_{2} \mathrm{PO}_{4}-$ $0.1 \mathrm{M} \mathrm{Na}_{2} \mathrm{HPO}_{4}, \mathrm{pH} 6.0,20 \%$ methanol, $500 \mathrm{mg} \mathrm{l}^{-1}$ sodium 1 -octanesulphonate and $50 \mathrm{mg}^{-1}$ disodium EDTA. The turnover rate of dopamine metabolite in the striatum was calculated by the equation of $[\mathrm{DA}]_{0}\left(\log [\mathrm{DA}]_{0}-\log [\mathrm{DA}]\right) /$ $0.434 \mathrm{t}, \mathrm{t}=4$, where $[\mathrm{DA}]_{0}$ and $[\mathrm{DA}]$ indicate dopamine contents in $0 \mathrm{mg}$ and $250 \mathrm{mg} \alpha$-methyl-p-tyrosine-treated groups, respectively (Carlsson et al., 1972).

\section{Materials}

Nebracetam was kindly given by Nippon Boehringer Ingelheim Co. Ltd. $\alpha$-Methyl-p-tyrosine and 3-hydroxybenzylhydrazine dihydrochloride were purchased from Sigma Chemical Co. (St. Louis, U.S.A.). All other reagents used were of the highest purity commercially available.

\section{Statistics}

The results are expressed as the means \pm s.e.mean. Statistical significance for comparison of the parameters between non- 
operated and microsphere-embolized groups was evaluated by Dunnett's multiple comparison test. Statistical significance between microsphere-embolized, nebracetam-treated microsphere-embolized, sham-operated and nebracetam-treated sham-operated groups, was by analysis of variance followed by Bonferroni's multiple comparison. Statistical significance in the in vivo monoamine synthesis and turnover rate between the untreated and treated groups was estimated by analysis of variance followed by Fisher's PLSD method. Differences with a probability of $5 \%$ or less were considered to be statistically significant $(P<0.05)$.

\section{Results}

\section{Changes in ACh and choline contents of rats with} microsphere embolism

In the first set of experiments, the effects of nebracetam on $\mathrm{ACh}$ and choline contents of the brain regions of the microsphere-embolism rats on the 3rd and 7th days after the operation were examined (Table 1). A significant decrease in ACh content was seen in the hippocampal region of the rats with microsphere embolism on both days after the embolism. Treatment with nebracetam did not reverse the decrease in the hippocampal ACh content.

Choline content of the three brain regions was also determined. The choline content in the striatum of the microsphere embolism rats increased only on the 3rd and 7th days, but not any other brain region and time examined after the embolism (data not shown). Treatment with nebracetam did not alter the embolism-induced increase in the striatal choline content.

\section{Changes in monoamine content of rats with microsphere embolism}

Changes in dopamine and its metabolites, DOPAC and HVA, were measured in the brain of microsphere embolism rats on the 3 rd and 7 th days after the operation. The results are shown in Figures 1, 2 and 3, respectively. A significant decrease in dopamine content was seen in the cerebral cortex on the 3rd day and in the striatum and hippocampus on the 3rd and 7th days after the embolism (Figure 1). Treatment with nebracetam did not reserve the decreased dopamine content at any brain region and time examined after the embolism. A significant decrease in DOPAC content was seen in the striatum on the 3 rd and 7 th days and in the hippocampus on the 7 th day after the embolism (Figure 2). HVA content of the three brain regions was significantly decreased on both days after the embolism (Figure 3). Delayed treatment with nebracetam significantly restored both DOPAC and HVA contents in the striatum on the 3rd day.

NA content of the three brain regions was also decreased after the embolism except for that of the cerebral cortex on the 7 th day (Table 1). Treatment did not affect the decreased NA content of any brain region and time examined after the embolism.

5-HT content was decreased in all brain regions examined on the 3rd and 7th days after the operation (Figure 4). A significant recovery of 5-HT content after treatment with nebracetam was seen only in the hippocampus on the 3rd day. The levels of 5-HIAA of the animals with microsphereembolism on the 3rd day were higher than baseline levels of the non-operated animals (Figure 5). This higher level was not reversed, but futher elevated, by treatment with nebracetam.

\section{Changes in in vivo hydroxylation of tyrosine and tryptophan}

Since appreciable effects of nebracetam treatment were seen in the striatal dopamine metabolites and in the hippocampal 5-HT and its metabolite, the second set of experiments was designed to focus further on alterations in these anabolic and catabolic pathways. At first, the ex vivo effect of nebracetam on the striatal dopamine and hippocampal 5-HT syntheses were determined in the untreated and nebracetam-

Table 1 Acetylcholine and noradrenaline contents of the cerebral cortex, striatum and hippocampus of the right hemisphere of rats with and without nebracetam treatment on the $3 \mathrm{rd}$ and 7 th days after micosphere embolism

\begin{tabular}{|c|c|c|c|c|c|}
\hline & \multirow{3}{*}{0 day } & \multicolumn{4}{|c|}{ Time after operation } \\
\hline & & \multicolumn{2}{|c|}{$3 r d d a y$} & \multicolumn{2}{|c|}{ 7th day } \\
\hline & & Untreated & Nebracetam & Untreated & Nebracetum \\
\hline \multicolumn{6}{|c|}{ Acetylcholine } \\
\hline \multicolumn{6}{|c|}{ Cortex } \\
\hline Pre & $20.1 \pm 1.2$ & & & & \\
\hline Sham & & $21.2 \pm 2.4$ & $19.8 \pm 1.6$ & $22.8 \pm 1.4$ & $23.8 \pm 1.0$ \\
\hline ME & & $16.1 \pm 1.6$ & $12.1 \pm 1.7$ & $17.9 \pm 2.1$ & $16.7 \pm 1.6$ \\
\hline \multicolumn{6}{|l|}{ Striatum } \\
\hline Pre & $83.2 \pm 3.2$ & & & & \\
\hline Sham & & $85.8 \pm 4.7$ & $82.9 \pm 3.4$ & $83.7 \pm 3.7$ & $82.0 \pm 1.7$ \\
\hline ME & & $50.3 \pm 13.1$ & $43.0 \pm 8.7$ & $60.2 \pm 8.3$ & $60.3 \pm 6.2$ \\
\hline \multicolumn{6}{|c|}{ Hippocampus } \\
\hline Pre & $35.2 \pm 1.2$ & & & & \\
\hline Sham & & $31.1 \pm 1.0$ & $31.9 \pm 0.9$ & $33.2 \pm 0.7$ & $34.4 \pm 1.4$ \\
\hline ME & & $17.9 \pm 1.7 \#$ & $16.7 \pm 1.7$ & $17.9 \pm 1.4 \#$ & $19.7 \pm 1.7$ \\
\hline \multicolumn{6}{|c|}{ Noradrenaline } \\
\hline \multicolumn{6}{|l|}{ Cortex } \\
\hline Pre & $1.55 \pm 0.26$ & & & & \\
\hline Sham & & $1.26 \pm 0.18$ & $2.38 \pm 0.61$ & $1.97 \pm 0.29$ & $1.36 \pm 0.12$ \\
\hline ME & & $0.73 \pm 0.26 \#$ & $0.90 \pm 0.13$ & $0.88 \pm 0.23$ & $0.66 \pm 0.12$ \\
\hline \multicolumn{6}{|l|}{ Striatum } \\
\hline Pre & $2.86 \pm 0.62$ & & & & \\
\hline Sham & & $1.85 \pm 0.41$ & $3.33 \pm 0.43$ & $3.20 \pm 0.66$ & $2.34 \pm 0.31$ \\
\hline ME & & $1.05 \pm 0.38 \#$ & $0.88 \pm 0.12$ & $1.46 \pm 0.14$ & $1.27 \pm 0.29$ \\
\hline \multicolumn{6}{|c|}{ Hippocampus } \\
\hline Pre & $3.50 \pm 0.99$ & & & & \\
\hline Sham & & $2.49 \pm 0.47$ & $3.15 \pm 0.33$ & $2.76 \pm 0.36$ & $2.21 \pm 0.13$ \\
\hline $\mathrm{ME}$ & & $0.73 \pm 0.27 \#$ & $1.29 \pm 0.27$ & $1.47 \pm 0.27 \#$ & $1.25 \pm 0.18$ \\
\hline
\end{tabular}

Values are expressed as nmol ${ }^{-1}$ frozen tissue. Each value represents the mean \pm s.e.mean of 6 to 8 experiments. \#Significantly different from the non-operated group (Pre) $(P<0.05)$. Abbreviations: Pre, non-operated group; Sham, sham-operated group; ME, group with microsphere embolism. 


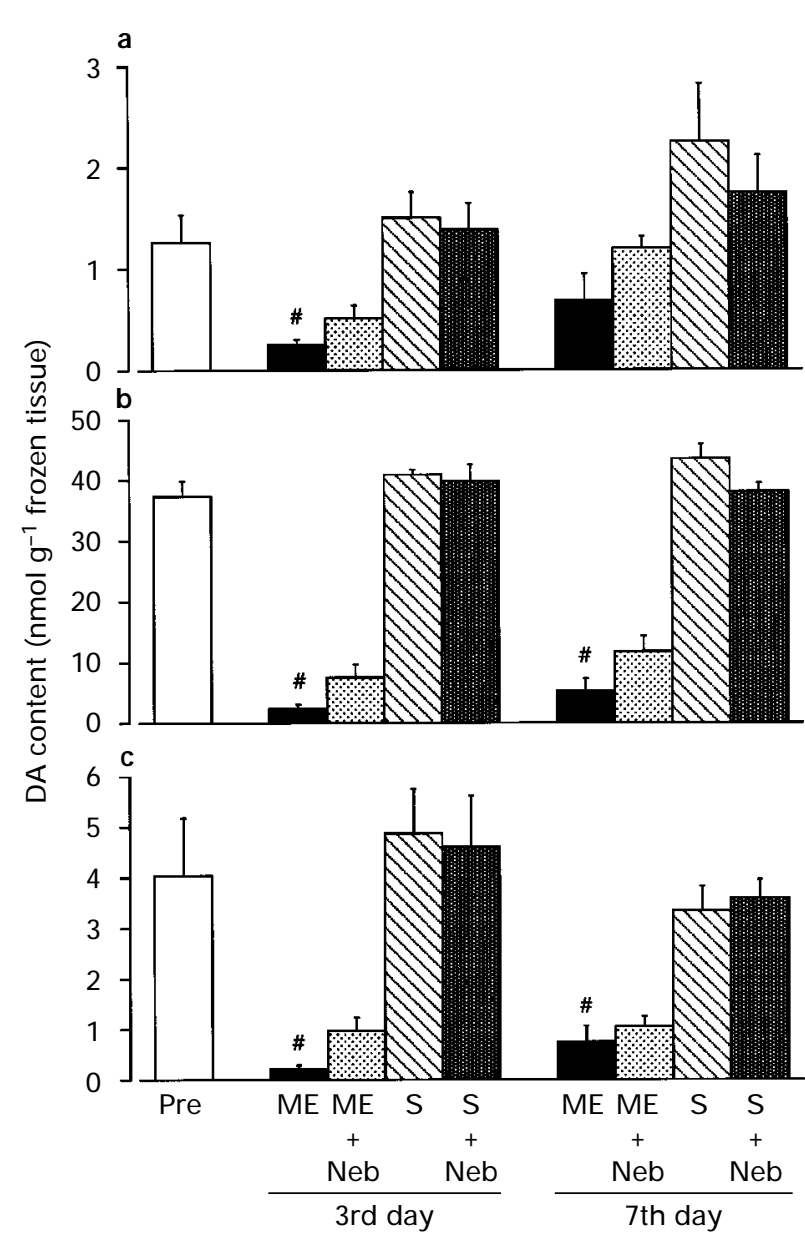

Figure 1 Changes in dopamine (DA) content of the (a) cerebral cortex, (b) striatum and (c) hippocampus of the right hemisphere of the non-operated rat (Pre), the untreated (ME) and nebracetamtreated rats with microsphere embolism $(\mathrm{ME}+\mathrm{Neb})$, and the untreated $(\mathrm{S})$ and nebracetam-treated sham-operated $(\mathrm{S}+\mathrm{Neb})$ rats on the $3 \mathrm{rd}$ and 7 th days after the operation. Each value represents the mean \pm s.e.mean of 6 to 8 experiments. The values for DA of the cerebral cortex, striatum and hippocampus of the non-operated rat were $1.26 \pm 0.27,37.31 \pm 2.51$ and $3.53 \pm 1.11 \mathrm{nmol} \mathrm{g}^{-1}$ frozen tissue $(n=7-8)$, respectively. \#Significantly different from the non-operated group $(P<0.05)$.

treated, microsphere embolism and sham-operated rats. For this purpose, the in vivo hydroxylation of tyrosine or tryptophan was determined in these animals on the 3rd day after the operation (Figure 6). There was no change in the striatal dopa and hippocampal 5-HTP accumulation of the sham-operated rats irrespective of treatment with nebracetam. Microsphere embolism resulted in a marked reduction in dopa and 5-HTP accumulation after inhibition of the hydroxylation. The ex vivo treatment of the microsphereembolized rat with nebracetam reversed the hippocampal 5-HTP accumulation, but not the striatal dopa accumulation.

\section{Changes in in vivo dopamine turnover}

In the third set of experiments, the in vivo dopamine turnover (dopamine catabolism) rate in the striatum was determined in the untreated and nebracetam-treated rats, rats with microsphere embolism and sham-operated rats on the 3rd day after the operation (Figure 7). This was examined because of the lack of an effect of ex vivo treatment with nebracetam on dopamine biosynthesis (Figure 6) and an appreciable effect on the striatal dopamine metabolites (Figures 2 and 3). The dopamine turn-

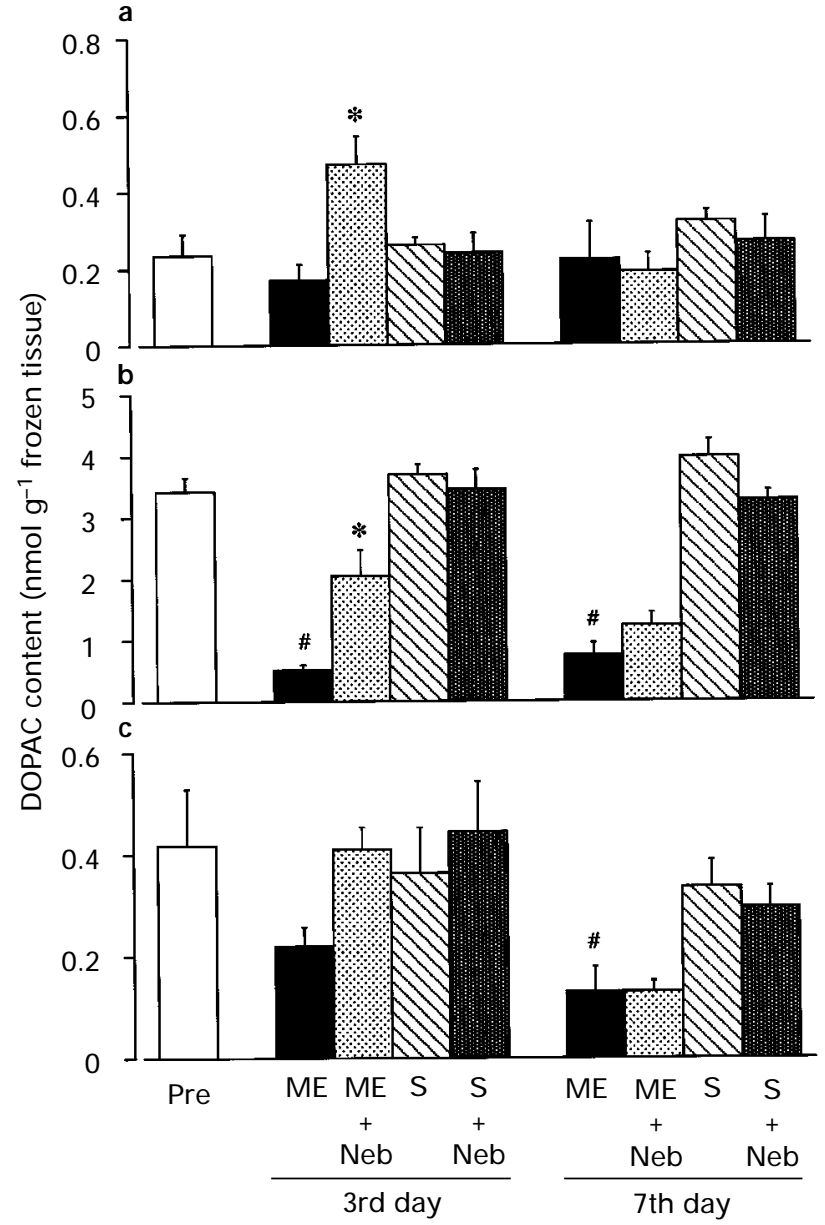

Figure 2 Changes in 3,4-dihydroxyphenylacetic acid (DOPAC) content of the (a) cerebral cortex, (b) striatum and (c) hippocampus of the right hemisphere of the non-operated rat (Pre), the untreated (ME) and nebracetam-treated rats with microsphere embolism $(\mathrm{ME}+\mathrm{Neb})$, and the untreated (S) and nebracetam-treated shamoperated rats $(\mathrm{S}+\mathrm{Neb})$ on the $3 \mathrm{rd}$ and 7 th days after the operation. Each value represents the mean \pm s.e.mean of 6 to 8 experiments. The values for DOPAC of the cerebral cortex, striatum and hippocampus of the non-operated rat were $0.24 \pm 0.05,3.42 \pm 0.23$ and $0.42 \pm 0.11 \mathrm{nmol} \mathrm{g}^{-1}$ frozen tissue $(n=8)$, respectively. \#Significantly different from the non-operated group and *significantly different from the corresponding untreated group $(P<0.05)$.

over rate was increased in the sham-operated rat by ex vivo treatment with nebracetam. A reduction in the striatal dopamine turnover rate was seen in rats after microsphere embolism irrespective of treatment with nebracetam.

\section{Discussion}

In the present study we aimed to elucidate the effects of delayed treatment with nebracetam on microsphere embolisminduced damage to cerebral neurotransmitter metabolism. Microsphere embolism decreased ACh content to a lesser degree than dopamine, NA and 5-HT contents in the present study. Furthermore, $\mathrm{ACh}$ and monoamine contents in the three brain regions of the animals with microsphere embolism on the 7th day were not different from those on the 3rd day after the embolism, suggesting that this microsphere embolism induced sustained or irreversible alterations in cerebral monoamine metabolism, rather than in ACh metabolism.

Although microsphere embolism resulted in a decreased ACh content in the hippocampus, the alteration was not affected by the delayed treatment with nebracetam. Nebracetam has been shown to enhance ACh synthesis and release in the 


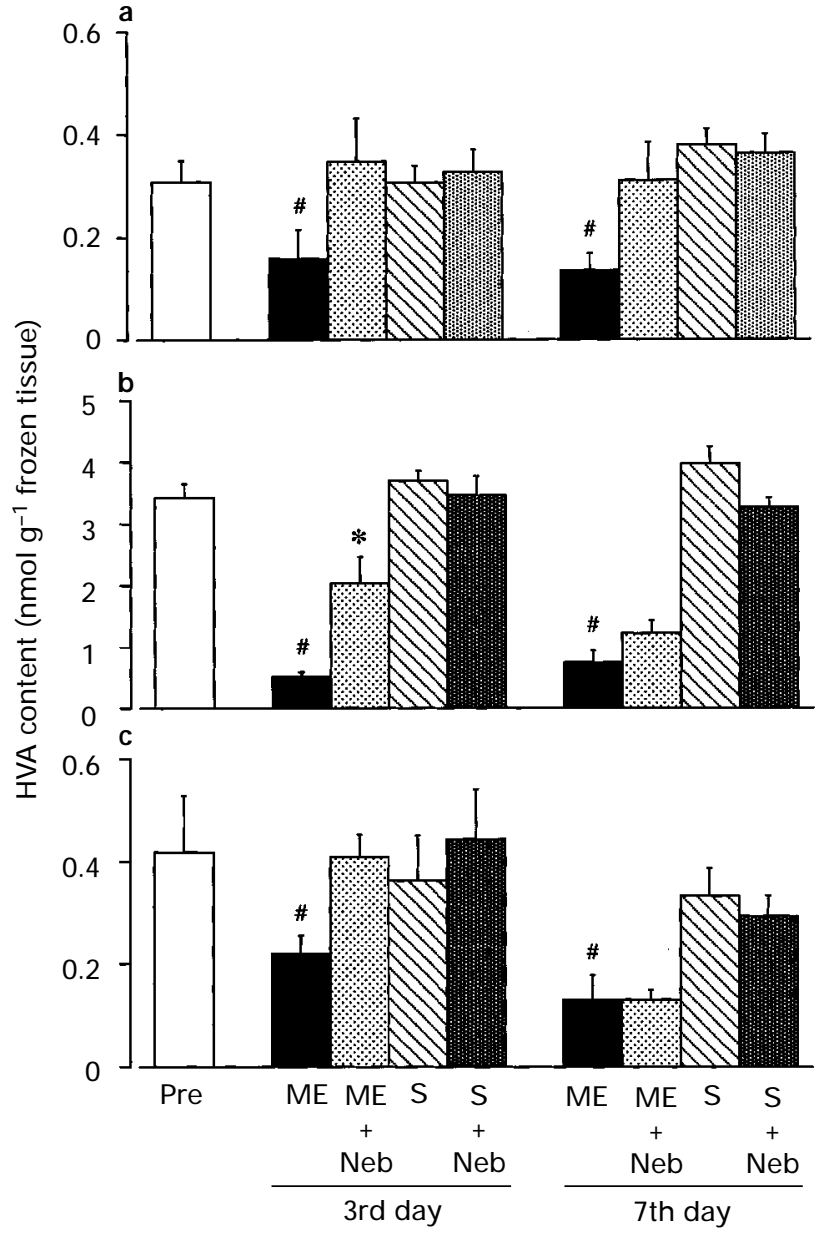

Figure 3 Changes in homovanillic acid (HVA) content of the (a) cerebral cortex, (b) striatum and (c) hippocampus of the right hemisphere of the non-operated rat (Pre), the untreated (ME) and nebracetam-treated rats with microsphere embolism $(\mathrm{ME}+\mathrm{Neb})$, and of the untreated (S) and nebracetam-treated sham-operated rats $(\mathrm{S}+\mathrm{Neb})$ on the $3 \mathrm{rd}$ and 7 th days after the operation. Each value represents the mean \pm s.e.mean of 6 to 8 experiments. The values for HVA of the cerebral cortex, striatum and hippocampus of the nonoperated rat were $0.31 \pm 0.04,2.47 \pm 0.15$ and $0.36 \pm 0.10 \mathrm{nmol} \mathrm{g}^{-1}$ frozen tissue $(n=8)$, respectively. \#Significantly different from the non-operated group and *significantly different from the corresponding untreated group $(P<0.05)$.

isolated ganglia of normal dogs (Ohjimi et al., 1994) and to prevent choline toxin-induced decrease in striatal ACh release and reduction in the striatal choline acetyltransferase activity of the rat (Hashimoto et al., 1991). The effects of the agent on the brain of animals compromised by hypoxia, ischaemia or ischaemia/reperfusion have not yet been elucidated. A binding study revealed that nebracetam has a weak affinity for muscarinic ACh receptors (Kitamura et al., 1990). In this respect, the finding that delayed treatment with nebracetam had no effect on ACh metabolism in the microsphere embolism-induced ischaemic brain was not unexpected.

A partial restoration of neurotransmitters in the ipsilateral hemisphere by delayed treatment with nebracetam was seen only with the striatal dopamine metabolite and the hippocampal 5-HT contents on the 3rd day after the embolism. As described above, alterations in monoamines, such as dopamine and 5-HT, were sustained much more than those in ACh after microsphere embolism. These findings motivated us to study further the mechanisms responsible for the restoration or conservation of neurotransmitter monoamines by nebracetam.

We examined the effects of delayed treatment with nebracetam on the biosynthesis of dopamine and 5-HT following

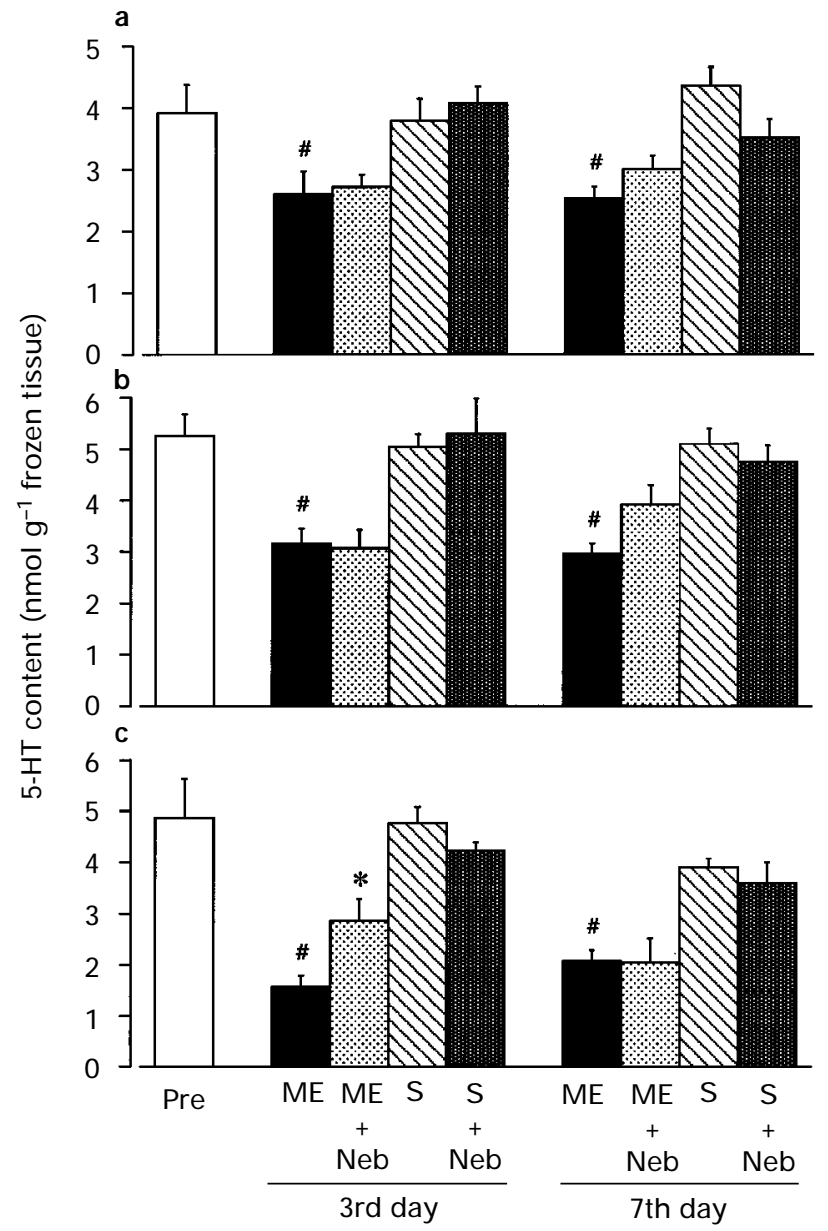

Figure 4 Changes in 5-hydroxytryptamine (5-HT) content of the (a) cerebral cortex, (b) striatum and (c) hippocampus of the right hemisphere of the non-operated rat (Pre), the untreated (ME) and nebracetam-treated rats with microsphere embolism $(\mathrm{ME}+\mathrm{Neb})$, and of the untreated $(\mathrm{S})$ and nebracetam-treated sham-operated rats $(\mathrm{S}+\mathrm{Neb})$ on the 3 rd and 7 th days after the operation. Each value represents the mean + s.e.mean of 6 to 8 experiments. The values for 5 -HT of the cerebral cortex, striatum and hippocampus of the nonoperated rat were $3.93+0.46,5.25+0.42$ and $4.26+0.90 \mathrm{nmol} \mathrm{g}^{-1}$ frozen tissue $(n=7-8)$, respectively. \#Significantly different from the non-operated group and *significantly different from the corresponding untreated group $(P<0.05)$.

microsphere embolism; that is, the effects of nebracetam on the in vivo dopa and 5-HTP accumulation in the striatum and hippocampus, respectively, were determined. Nebracetam did not alter the striatal dopa and hippocampal 5-HTP accumulation of the sham-operated animals following administration of the aromatic L-amino acid decarboxylase inhibitor, suggesting that nebracetam per se does not affect the synthesis of striatal dopamine and hippocampal 5-HT under normal conditions. The effect of delayed treatment with nebracetam on dopamine or 5-HT synthesis was detected only in the hippocampal tryptophan hydroxylation in the rats with microsphere-embolism. This suggests that 5-HT synthesis is protected against microsphere embolism-induced ischaemic injury or the impaired 5-HT synthesis is reversed by the delayed treatment with nebracetam.

Since no appreciable effects of nebracetam on the in vivo hydroxylation of tyrosine were detected, its effect on striatal dopamine catabolism following microsphere embolism was further examined under in vivo conditions. Although dopamine turnover rate was enhanced by delayed treatment with nebracetam in the sham-operated animal, such enhancement was not observed in the rats with microsphere-embolism. We 


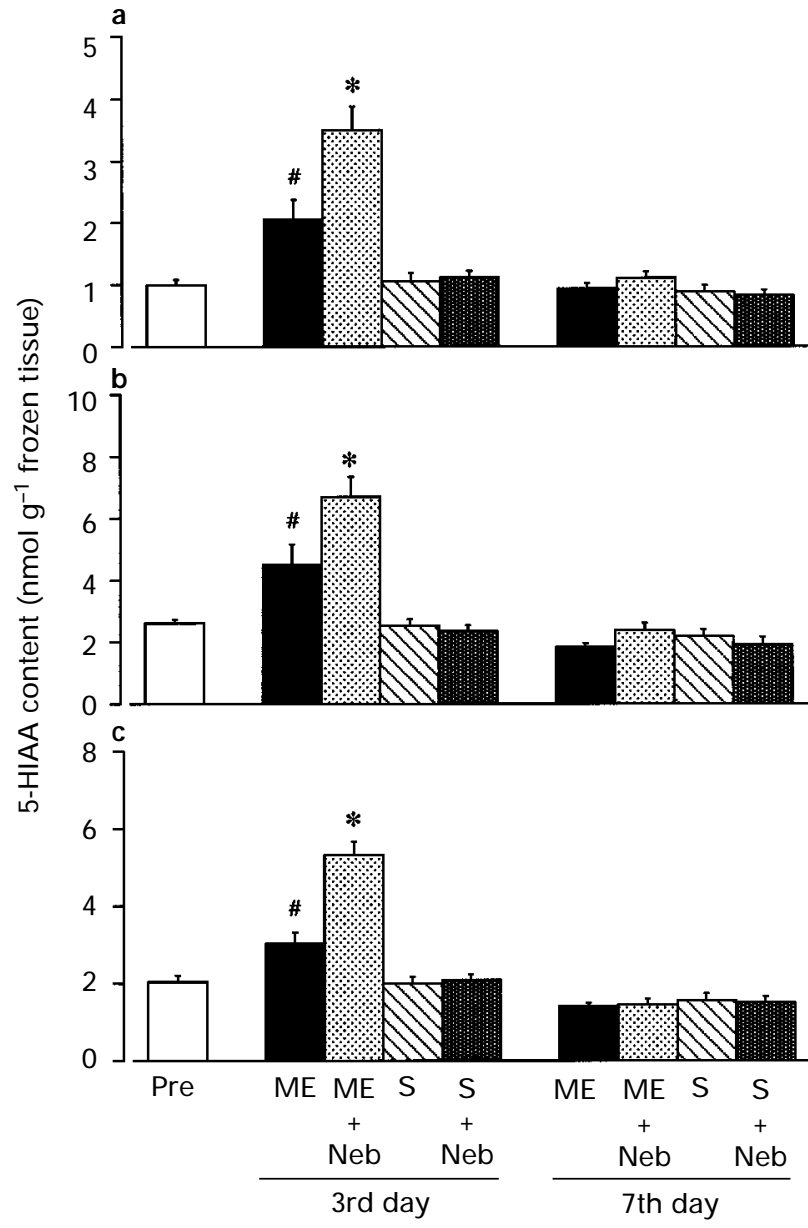

Figure 5 Changes in 5-hydroxyindoleacetic acid (5-HIAA) content of the (a) cerebral cortex, (b) striatum and (c) hippocampus of the right hemisphere of the non-operated rat (Pre), the untreated (ME) and nebracetam-treated rats with microsphere embolism (ME $+\mathrm{Neb})$, and the untreated $(\mathrm{S})$ and nebracetam-treated sham-operated rats $(\mathrm{S}+\mathrm{Neb})$ on the $3 \mathrm{rd}$ and 7 th days after the operation. Each value represents the mean \pm s.e.mean of 6 to 8 experiments. The values for 5-HIAA of the cerebral cortex, striatum and hippocampus of the non-operated rat were $1.00 \pm 0.08, \quad 2.61 \pm 0.12$ and $2.04 \pm$ $0.16 \mathrm{nmol} \mathrm{g}^{-1}$ frozen tissue $(n=8)$, respectively. \#Significantly different from the non-operated group and *significantly different from the corresponding untreated group $(P<0.05)$.

observed that the striatal DOPAC and HVA levels on the 3rd day were higher in the nebracetam-treated rats with microsphere-embolism than the untreated rats with microsphereembolism. The striatal dopamine levels were also higher in the drug-treated rats with microsphere embolism than in the untreated rats with microsphere embolism on the 3rd day (Figure 1). The absolute difference in the striatal dopamine metabolite levels in the untreated and treated rats with microsphere embolism appears to be comparable to the differences in dopamine levels per se between these two groups. This substantial difference in dopamine levels, rather than the effect on synthesis and/or catabolism of dopamine, may be at least in part attributed to the elevation of striatal DOPAC and HVA contents of the nebracetam-treated rats with microsphere embolism.

5-HIAA levels in the three brain regions of the animals with microsphere embolism on the 3rd day were greater than baseline levels of the non-operated animals and the delayed treatment with nebracetam further elevated the 5-HIAA levels. Since no effects of this treatment on 5-HIAA content were seen in the sham-operated animals on the 3rd day and in the animals with microsphere embolism on the 7 th day, the effects may be specific in the ischaemic brain on the 3rd day.
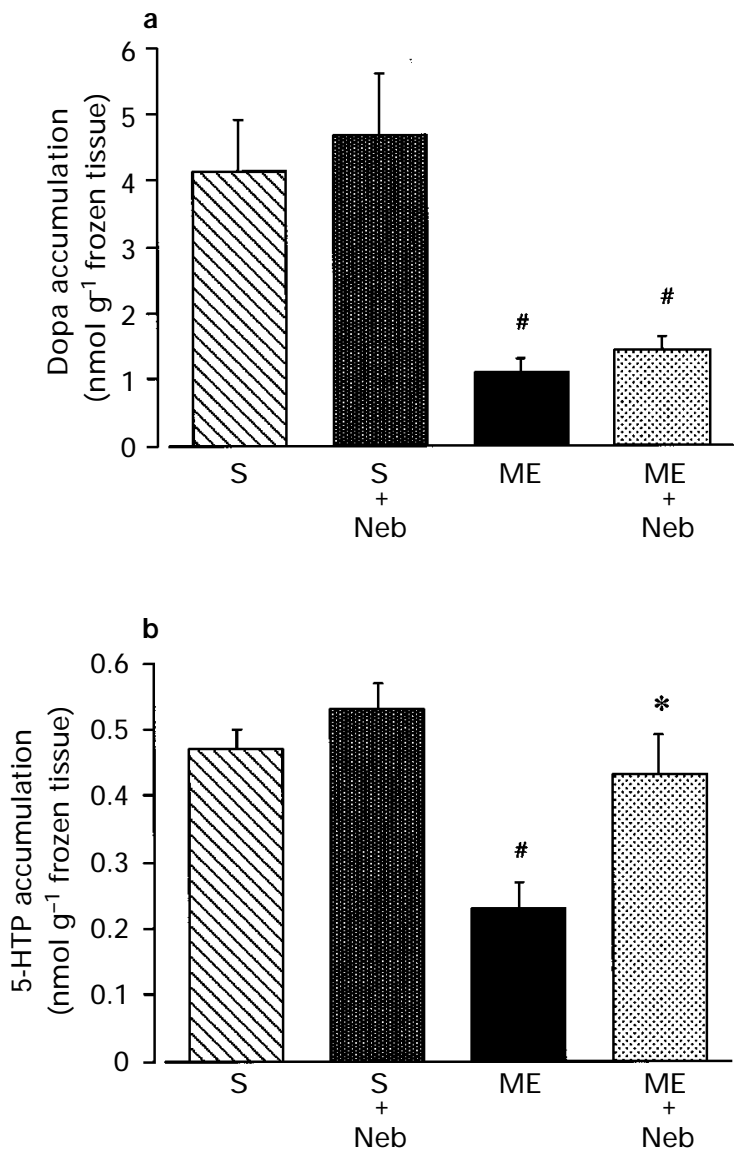

Figure 6 In vivo tyrosine and tryptophan hydroxylation in the striatum and hippocampus, respectively, of the right hemisphere of the untreated (S) and nebracetam-treated sham-operated rats $(\mathrm{S}+\mathrm{Neb})$, and the untreated $(\mathrm{ME})$ and treated rats with microsphere embolism $(\mathrm{ME}+\mathrm{Neb})$ on the 3rd day after the operation. Tyrosine hydroxylation was estimated by the accumulation of dopa in the striatum (a), whereas tryptophan hydroxylation, by the accumulation of 5-hydroxytryptophan (5-HTP) in the hippocampus (b) after in vivo treatment with an inhibitor of aromatic L-amino acid decarboxylase. Each value represents the mean \pm s.e.mean of 7 experiments. \#Significantly different from the corresponding sham-operated group and *significantly different from the untreated group $(P<0.05)$.

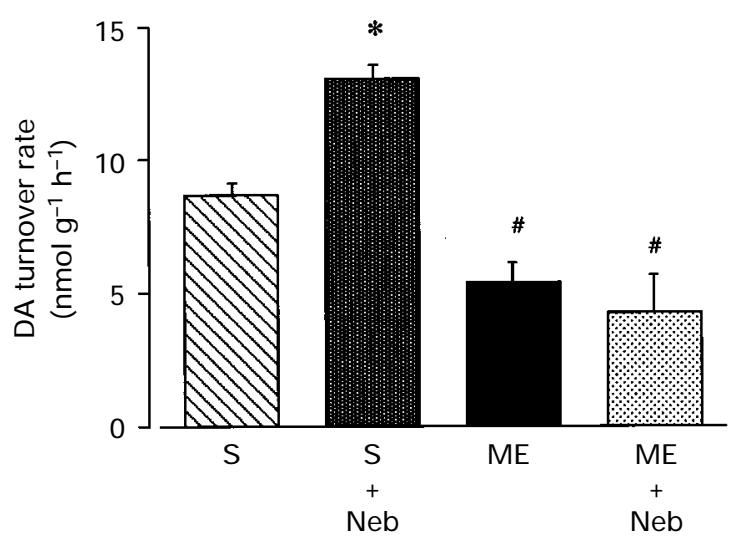

Figure 7 In vivo dopamine turnover rate in the striatum of the right hemisphere of the untreated (S) and nebracetam-treated shamoperated rats $(\mathrm{S}+\mathrm{Neb})$, untreated $(\mathrm{ME})$ and treated rats with microsphere embolism $(\mathrm{ME}+\mathrm{Neb})$ on the 3rd day after the operation. The striatal dopamine turnover rate was estimated by differences in the amounts of dopamine in the presence and absence of $\alpha$-methyl-p-tyrosine. Each value represents the mean \pm s.e.mean of 4 experiments. \#Significantly different from the corresponding shamoperated group and *significantly different from the untreated group $(P<0.05)$. 
Generally, there are several factors which determine changes in metabolites in a tissue, such as production and degradation of metabolites in the tissue and washout of metabolites from the tissue. A conclusive mechanism for alterations in the metabolite content can be proposed only after extensive examination of these determinants. With regard to 5-HIAA, 5-HT synthesis in the hippocampus was reduced in the rats with microsphere embolism on the 3rd day and the delayed treatment with nebracetam restored the hippocampal biosynthesis of 5-HT appreciably. These findings suggest that 5-HIAA levels are higher in the nebracetam-treated animals than the untreated animal, although we have no data concerning its effects on in vivo 5-HT turnover in the present study. Furthermore, the extent of washout of metabolites might be a possible factor to determine tissue metabolite content in the ischaemic brain. In a previous study, we observed a marked decrease in blood flow in all three brain regions on the $3 \mathrm{rd}$ day and moderate or lesser decreases in the blood flow in these brain regions on the 7 th day after microsphere embolism (Miyake et al., 1993). Thus, it is possible that reduced blood flow, that is a failure in washout of the metabolite, may contribute to the elevation of 5-HIAA levels in the brain of the rats with microsphere embolism irrespective of treatment with nebracetam. Since the cerebral blood flow of the rats with microsphere embolism with and without nebracetam treatment was not determined in the present study, the exact mechanism for the increase in 5-HIAA levels in the brain remains inconclusive at present.

It is recognized that cerebral cholinergic nerve activity plays a pivotal role in learning and memory function (Bartus et al., 1982; Hargan \& Morris, 1987). In fact, the learning and memory deficits of dementia in the aged and patients with Alzheimer's disease have been associated with a decline in cholinergic systems of the basal forebrain (Drachman \& Lea- vitt, 1974; Coyle et al., 1983). This suggests a possible relationship between deficits in learning and memory function and impairments in central cholinergic systems under pathophysiological conditions. On the basis of this hypothesis, it is possible that changes in $\mathrm{ACh}$ metabolism in the ischaemic brain may be, at least in part, related to nootropic effects of an agent. However, we did not observe any effects of nebracetam treatment on cerebral ACh content in the rats with microsphere-embolism. Thus, the contribution of ACh metabolites to a putative nootropic action of nebracetam in the rats with microsphere embolism is unlikely.

Since there is so far no evidence for a definite relationship between the 5-hydroxytryptaminergic system and learning and memory, the contribution of 5-HT metabolism modified by nebracetam to the learning and memory function would be speculative. Several studies have predicted an interaction between the 5-hydroxytryptaminergic and cholinergic systems in mediating the learning and memory processes. That is, Nilsson et al. (1988) showed that a combination of cholinergic and 5-hydroxytryptaminergic denervation of the rat forebrain produced profound impairments in spatial learning of rats, and Riekkinen et al. (1990) observed that a dihydroxytryptamine-induced lesion of raphe dorsalis aggravated the spatial navigation deficit produced by a lesion of the nucleus basalis magnocellularis in rats. These observations suggest that the 5-hydroxytryptaminergic system plays a potential role in learning and memory. If so, restoration of 5-HT metabolism by nebracetam might play a role in learning and memory under ischaemic conditions.

In summary, in the present study we demonstrated that nebracetam enhanced 5-HT synthesis in the hippocampus of rats with microsphere embolism. Although the significance of the effects remains unclear, the results provide evidence for a possible action of nebracetam on restoration of hippocampal 5-HT synthesis in the ischaemic brain.

\section{References}

BARTUS, R.T., DEAN, R.L. III, BEER, B. \& LIPPA, A.S. (1982). The cholinergic hypothesis of geriatric memory dysfunction. Science, 217, $408-417$.

CARLSSON, A., DAVIS, J.N., KEHR, W., LINDQUIST, M. \& ATACK, C.V. (1972). Simultaneous measurement of tyrosine and tryptophan hydroxylase activities in brain in vivo using an inhibitor of the aromatic amino acid decarboxylase. Naunyn-Schmiedeberg's Arch. Pharmacol., 275, $153-168$.

COYLE, J.T., PRICE, D.J. \& DELONG, M.R. (1983). Alzheimer's Disease: A disorder of cortical cholinergic innervation. Science, 219, $1184-1190$.

DRACHMAN, D.A. \& LEAVITT, J. (1974). Human memory and the cholinergic system - a relationship to aging? Arch. Neurol., 30, $113-121$.

FURLOW, T. \& BASS, N.H. (1976). Arachidonate-induced cerebrovascular occlusion in the rat. Neurology, 26, 297-304.

HARGAN, J.J. \& MORRIS, R.G.M. (1987). The cholinergic hypothesis of memory: a review of animal experiments. In The Handbook of Psychopharmacology, ed. Synder, S., Iversen, L.L. \& Iversen, S.D. pp. 237-323, New York: Plenum Press.

HASHIMOTO, M., HASHIMOTO, T. \& KURIYAMA, K. (1991). Protective effect of WEB 1881 FU on AF64A (ethylcholine aziridinium ion)-induced impairment of hippocampal cholinergic neurons and learning acquisition. Eur. J. Pharmacol., 209, 9-14.

IWASAKI, K., MATSUMOTO, Y. \& FUJIWARA, M. (1992). Effect of nebracetam on the disruption of spatial cognition in rats. Jpn. J. Pharmacol., 58, $117-126$.

KITAMURA, Y., HAYASHI, S. \& NOMURA, Y. (1990). Effects of WEB 1881 FU, a novel nootropic, on cholinergic and adrenergic receptors in the rat brain: Action on $\mathbf{M}_{1}$-muscarinic receptors. Jpn. J. Pharmacol., 52, 597-607.

KUHN, F.J., SCHINGNITS, G., LEHR, E., MONTAGNA, E., HINZEN, H.D. \& GIACHETTI, A. (1988). Pharmacology of WEB 1881-FU, a central cholinergic agent, which enhances cognition and cerebral metabolism. Arch. Int. Pharmacodyn. Ther., 292, 13-34.

MCGRAW, C.P. (1977). Experimental cerebral infarction: effect of pentobarbital in mongolian gerbils. Arch. Neurol., 34, 334-336.

MIYAKE, K., TAKEO, S. \& KAJIHARA, H. (1993). Sustained decrease in brain regional blood flow after microsphere embolism in rats. Stroke, 24, 415-420.

MIYAKE, K., TANONAKA, K., MINEMATSU, R., INOUE, K. \& TAKEO, S. (1989). Possible therapeutic effect of naftidrofuryl oxalate on brain energy metabolism after microsphere-induced cerebral embolism. Br. J. Pharmacol., 98, 389-396.

NILSSON, O.G., STRECHER, R.E., DASZUTA, A. \& BJORKLUND, A (1988). Combined cholinergic and serotonergic denervation of the forebrain produces severe deficits in a spatial learning task in the rat. Brain Res., 453, $235-246$.

OHJIMI, H., KUSHIKU, K., YAMADA, H., KUWAHARA, T, KOHNO, Y. \& FURUKAWA, T. (1994). Increase of acetylcholine release by nebracetam in dog cardiac synaptic ganglion. J. Pharmacol. Exp. Ther., 268, 396-402.

OHTOMO, E., KUTUKAKE, N., HASEGAWA, K., HIRAI, S., KUZUYA, F., NISHIMURA, K., FUJISHIMA, M., INANAGA, K. \& NAKASHIMA, M. (1992). Clinical evaluation of WEB 1881 (Nebracetam fumarate) in patients with cerebrovascular disorders. - A double blind study in comparison with idebenone. Rinsho Iyaku, 8, $1063-1106$

RIEKINEN, Jr., P., SIRVIO, J. \& RIEKKINEN, P. (1990). Interaction between raphe dorsalis and nucleus basalis magnocellularis in spatial learning. Brain Res., 527, $342-345$.

SHIBATA, S., KODAMA, K., TOMINAGA, K., TANAKA, K. \& WATANABE, S. (1992). Effect of muscarinic cholinergic drugs on ischaemia-induced decrease in glucose uptake and CA1 field potentials in rat hippocampus slices. Eur. J. Pharmacol., 221, $113-119$. 
TAGUCHI, T., TAKAGI, N., MIYAKE, K., TANONAKA, K., OKADA M., KAJIHARA, H. \& TAKEO, S. (1994). Effects of naftidrofuryl oxalate on microsphere-induced changes in acetylcholine and amino acid content of rat brain regions. Exp. Brain Res., 99, $7-$ 16.

TAKAGI, N., MIYAKE, K., OHIWA, A., NUKAGA, R. \& TAKEO, S. (1996). Effects of delayed treatment with nafronyl oxalate on microsphere embolism-induced changes in monoamine levels of rat brain regions. Br. J. Pharmacol., 118, 33-40.

TAKAGI, N., TSURU, H., YAMAMURA, M. \& TAKEO, S. (1995). Changes in striatal dopamine metabolism after microsphere embolism in rats. Stroke, 26, $1101-1106$.
TAKEO, S., MIYAKE, K., TANONAKA, K., TAKAGI, N., TAKAGI, K., KISHIMOTO, K., SUZUKI, M., KATSURAGI, A., GOTO, M. \& OSHIKAWA, S. (1997). Beneficial effect of nebracetam on energy metabolism after microsphere-induced embolism in rat brain. Arch. Int. Pharmacodyn. Ther., 331, 232-245.

TAKEO, S., TAGUCHI, T., TANONAKA, K., MIYAKE, K., HORIGUCHI, T., TAKAGI, N. \& FUJIMORI, K. (1992). Sustained damage to energy metabolism of brain regions after microsphere embolism in rats. Stroke, 23, $62-68$.

Received November 18, 1996 Revised January 23, 1997 Accepted February 28, 1997 